\title{
Prenatal diagnosis of partial absence of the tricuspid valve with conoventricular septal defect - The utility of prenatal 4-Dimensional Spatiotemporal Image Correlation Rendering.
} \author{
Syed $^{4}$, and Balaji Srimurugan ${ }^{1}$ \\ ${ }^{1}$ Amrita Institute of Medical Sciences and Research Centre \\ ${ }^{2}$ Lakshimi Madhavan hospital \\ ${ }^{3}$ Royal Hospital, \\ ${ }^{4}$ Royal hospital
}

Balaganesh Karmegaraj ${ }^{1}$, Madhubala Manickavasagam ${ }^{2}$, Syed Ibrahim ${ }^{3}$, Mohamed Razeen

January 11, 2021

\begin{abstract}
First described in 1965 by Johnson and Wilcox, partial absence of the tricuspid valve (TV) associated with a ventricular septal defect(VSD) is a very rare malformation.(1) Heart failure secondary to severe TV regurgitation results in-utero fetal compromise. (2) We present here a fetus with partial absence of the tricuspid valve with conoventricular septal defect.
\end{abstract}

\section{Case report:}

A 26-year-old primigravida was referred for fetal echocardiography at 28 weeks gestation in view of suspicion of pulmonary stenosis and VSD on screening ultrasound. Fetal cardiac screening preformed at first and second trimester were reported normal. The apical 4-chamber view demonstrated dilated right atrium and to and fro flow across the TV. There was low pressure tricuspid regurgitation (TV PGmax 3.9mmHg). [Figure 1; Movie 1] The lateral 4-chamber view showed normal offsetting of the atrioventricular (AV) valves and the anterior tricuspid valve leaflet (ATL) was inserted to the anterior papillary muscle.[Movie 2] The 4chamber view continuous sweep from posterior to anterior showed absent posterior tricuspid leaflet and partially deficient ATL. [Figure 2; Movie 3] Left ventricular outflow tract view showed a large VSD particularly restricted by thickened septal tricuspid leaflet (STL).[Figure 3; Movie 4 and 5] High short axis view of the fetal heart showed a large VSD in the conoventricular region.[Figure 4; Movie 6] The outflow tracts were normally related and the main pulmonary artery was smaller [MPA $4.6 \mathrm{~mm}$ ( $\mathrm{Z}$ score -2.4)] than the ascending aorta. There was antegrade flow across the pulmonary valve (Vmax $0.72 \mathrm{~m} / \mathrm{s})$. Low short axis view at AV valves showed a normal mitral valve(MV) and a tricuspid valve (TV) with absent posterior tricuspid leaflet [PTL].[Figure 5A; Movie 7] Parasternal long axis right ventricle inflow view showed absent PTL, deficient ATL and color Doppler demonstrated free TR. [Figure 5B and 5C; Movie 8] Four dimensional images were obtained using volume transducer probe CV 1-8A [Samsung W10 equipment]. 4-dimensional high definition transverse surface rendering of the fetal heart slightly above the level of AV septum revealed the anatomy of both the AV valves. The MV was normal and there was non coaptation of TV. The TV was dysplastic with completely absent PTL and deficient ATL. [Figure 6A-C; Movie 9]

\section{In-utero course:}

Followup echocardiography at 34 weeks gestation revealed hydrops fetalis, severe right atrial dilatation, RV dysfunction, bradycardia $(80 \mathrm{bpm})$ and absent end diastolic velocity in the umbilical arteries.[Figure 7A 
and B] The fetus was died in-utero. A 2000g male baby was delivered with absent cardiac activity through normal delivery. Option of fetal autopsy and genetic evaluation of the fetal intracardiac blood were given but parents denied further evaluation. A schematic cartoon demonstrating the surgeons view of this anomaly after opening the right atrium is shown in the Figure 8.

\section{Conclusion:}

Tricuspid regurgitation is the hallmark finding in Ebstein's anomaly and other tricuspid valve disorders, whereas in partial absence of TV leaflets results in 'to and fro' flow across the right sided chambers. Severe TV regurgitation leads to compromised forward flow in to the pulmonary artery resulted in smaller main pulmonary artery in this case. The Color Doppler flow pattern helps in differentiating this anomaly from others. Hence precise sequential segmental evaluation of the fetal heart is of significant importance. Fourdimensional spatiotemporal image correlation rendering provides incremental benefit in evaluation of TV anomalies. This will help in counselling parents about the expected pregnancy outcome.
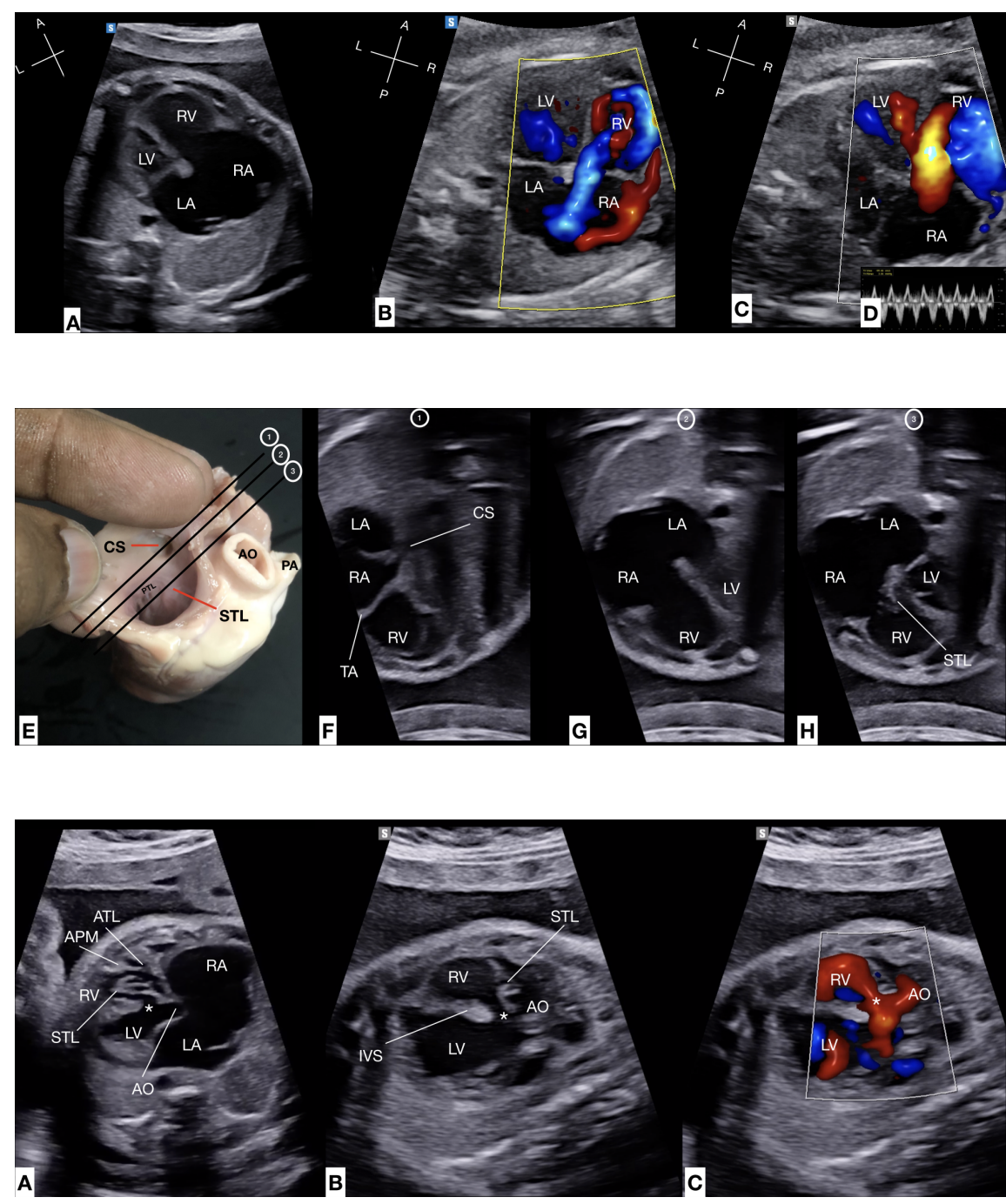

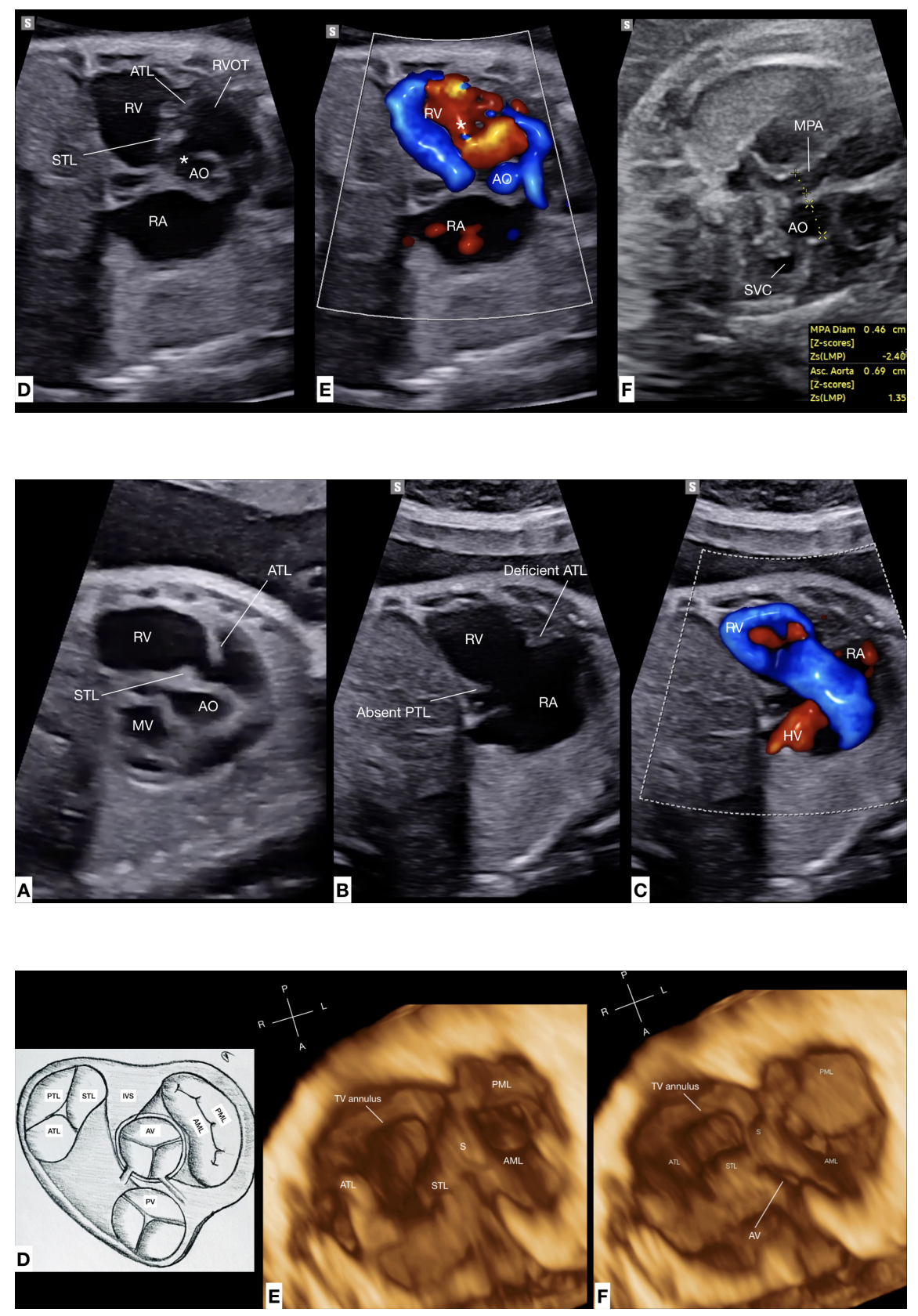

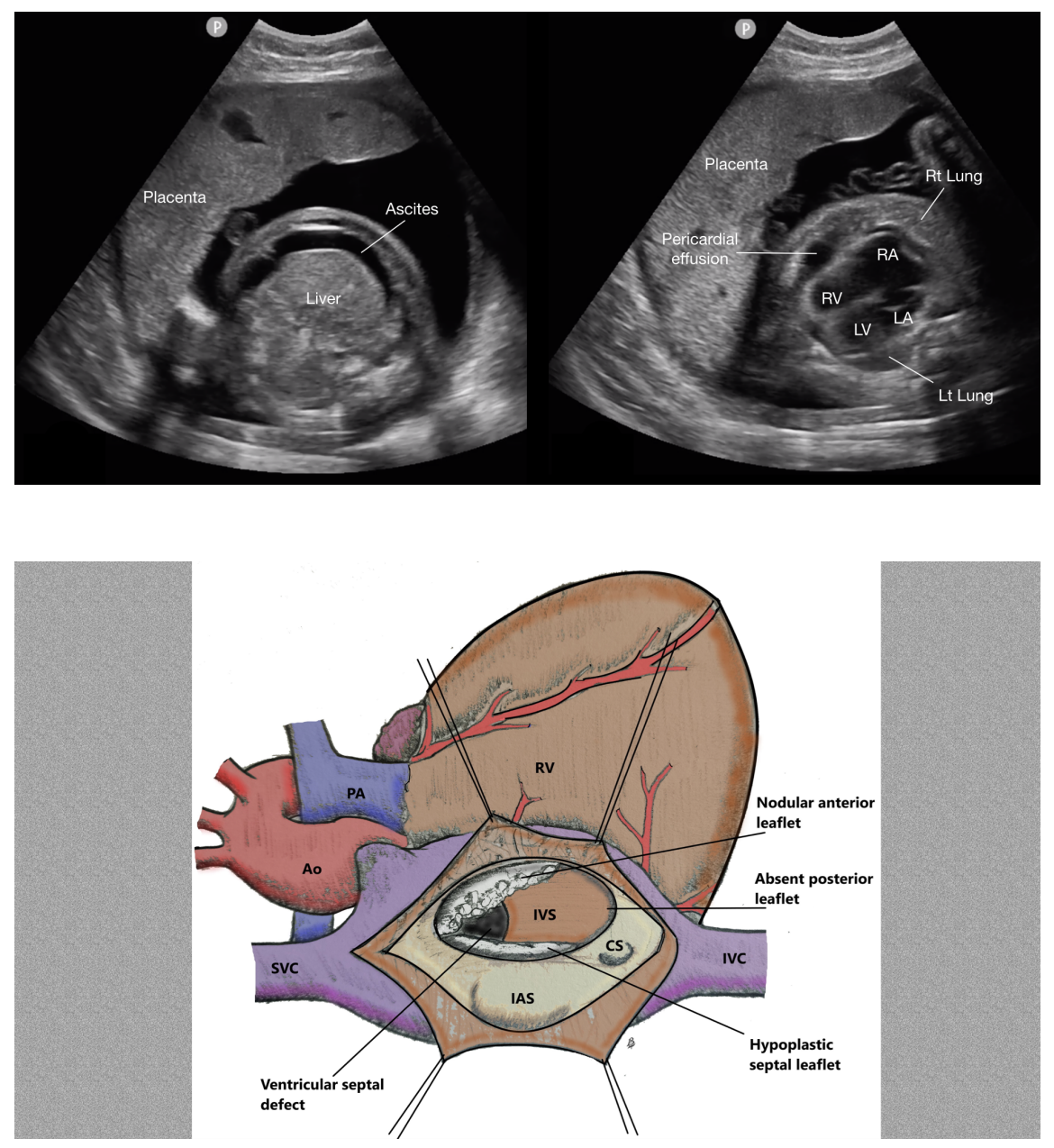